\title{
Patients with Gout Treated with Conventional Urate-lowering Therapy: Association with Disease Control, Health-related Quality of Life, and Work Productivity
}

\author{
Robert Wood, Steve Fermer, Sulabha Ramachandran, Scott Baumgartner, and Robert Morlock
}

\begin{abstract}
Objective. Implications of inadequate gout control were assessed through health-related quality of life (HRQOL) and work productivity of patients with gout adequately controlled while taking conventional urate-lowering therapy (ULT) for $\geq 3$ months vs those whose gout was inadequately controlled.

Methods. Retrospective data were drawn from the Adelphi Disease Specific Programme (DSP), a cross-sectional survey of patients with gout in France, Germany, the United Kingdom, and the United States. Patients completed these questionnaires: EQ-5D (3L), Patient Reported Outcomes Measurement Information System (PROMIS) Health Assessment Questionnaire (HAQ), and Work Productivity and Activity Impairment. Inadequate control was defined as the most recent serum uric acid (SUA) level $>6 \mathrm{mg} / \mathrm{dl}(>360 \mu \mathrm{mol} / \mathrm{l})$ or $\geq 2$ flares in the last 12 months; adequate control as SUA level $\leq 6 \mathrm{mg} / \mathrm{dl}(\leq 360 \mu \mathrm{mol} / \mathrm{l})$ and 0 flares. Appropriate statistical tests were used to assess differences between groups.

Results. There were 836 (69\%) inadequately and 368 (31\%) adequately controlled gout cases. Mean age was 61 and 63 years and duration of current ULT was 32 and 57 months, respectively. Patients experiencing inadequate control reported significantly worse functioning and HRQOL, as measured by the EQ-5D (0.790 vs 0.877 ; difference: -0.087 ; $\mathrm{p}<0.001)$ and PROMIS HAQ (13.21 vs 6.91; difference: $6.30 ; \mathrm{p}<0.001$ ) scales. Productivity was also more impaired (work time missed: $4.5 \%$ vs $1.3 \%$; impairment while working: $19.1 \%$ vs $5.2 \%$; overall work impairment: $20.4 \%$ vs $5.6 \%$; activity impairment: $20.3 \%$ vs $5.3 \%$; all p < 0.001).

Conclusion. Less than one-third of patients had gout that was adequately controlled. Those experiencing inadequately controlled gout reported significantly worse functioning, quality of life, and work productivity. Gout treatment strategies to improve disease control may lead to improvements in HRQOL and productivity. (First Release April 1 2016; J Rheumatol 2016;43:1897-903; doi:10.3899/ jrheum.151199)
\end{abstract}

Key Indexing Terms:

GOUT

URATE-LOWERING THERAPY

Gout, the most common form of inflammatory arthritis ${ }^{1}$, is caused by hyperuricemia [typically defined as serum uric acid (SUA) $>6.8 \mathrm{mg} / \mathrm{dl}(408 \mu \mathrm{mol} / \mathrm{l})]$. The amount of uric acid in the body depends on endogenous synthesis, dietary intake of purines, and excretion. While diet and overproduction of uric acid may contribute to hyperuricemia (10\%), it is usually because of inefficient uric acid excretion $(90 \%)^{2}$. Some individuals may have both an overproduction and an

From Adelphi Real World, Cheshire, UK; AstraZeneca, Wilmington, Delaware; Ardea Biosciences Inc., San Diego, California, USA.

R. Wood, BSc, Adelphi Real World; S. Fermer, Adelphi Real World; S. Ramachandran, PhD, former employee, AstraZeneca; S. Baumgartner, MD, Ardea Biosciences Inc.; R. Morlock, PhD, Ardea Biosciences Inc.

Editorial support funded by AstraZeneca.

Address correspondence to R. Wood, Adelphi Real World, Adelphi Mill, Macclesfield, Cheshire SK10 5JB, UK.

E-mail:Robert.Wood@adelphigroup.com

Full Release Article. For details see Reprints/Permissions at jrheum.org Accepted for publication February 23, 2016.

\section{HEALTH-RELATED QUALITY OF LIFE WORK PRODUCTIVITY}

inefficient excretion of uric acid $^{3}$. Longterm hyperuricemia can lead to urate crystal deposition disease, with deposits of monosodium urate crystals in musculoskeletal structures and other tissues, causing chronic inflammation, acute gout flares, and potentially chronic arthritis with joint damage and disfiguring tophi ${ }^{4}$. Both hyperuricemia and gout are associated with comorbidities such as hypertension, cardiovascular disease, kidney disease, and metabolic syndrome, including diabetes ${ }^{5}$.

In addition to causing pain, disability, and diminished quality of life, poorly controlled gout is associated with significantly higher healthcare costs and losses in productivity $6,7,8,9$. Recently, annual medical care costs for gout in the United States were conservatively estimated to exceed $\$ 6$ billion in direct and indirect costs based on then-available prevalence data ${ }^{1}$.

The longterm treatment goal for gout is to reduce SUA levels to $<6 \mathrm{mg} / \mathrm{dl}(<360 \mu \mathrm{mol} / \mathrm{l})$ to prevent new crystal

Personal non-commercial use only. The Journal of Rheumatology Copyright @ 2016. All rights reserved. 
formation and dissolve existing crystals ${ }^{10}$. However, patients treated with conventional urate-lowering therapy (ULT) often have gout that is inadequately controlled ${ }^{11}$. As a result of frequent suboptimal treatment as well as excruciating pain, chronic arthropathy, and associated comorbidities, health-related quality of life (HRQOL) is impaired in patients with gout ${ }^{12}$. Patients with gout are also less productive at work, experience activity limitations, and use more healthcare resources than people without gout ${ }^{13,14,15}$.

Treatment guidelines and strategies for chronic diseases such as gout are often based on results from randomized controlled trials. However, these results may have limited relevance to real-world clinical practice because of the inclusion/exclusion criteria for patients included in trials. Noninterventional observational studies, with their wider patient base, may overcome some of the inherent challenges to generalizing the results from randomized trials and may provide a link from such trials to real-world clinical practice. However, these types of longitudinal studies do not effectively identify the attitudes and expectations that motivate patients to seek treatment and physicians to prescribe treatment. We used a cross-sectional analysis of physician-recorded demographic and clinical data to assess HRQOL, work productivity, and activity limitations of patients with gout that was inadequately controlled with conventional ULT for $\geq 3$ months compared with gout that was adequately controlled.

\section{MATERIALS AND METHODS}

Study design and population. This was a cross-sectional, retrospective analysis of data obtained from the Adelphi Real World (Macclesfield, Cheshire, UK) Disease Specific Program (DSP) for gout, a multinational, real-world survey of patients with gout who consulted practicing rheumatologists and primary care physicians. The DSP was designed to collect a cross-section of data that reflect patient attitudes and associated physician treatment practices ${ }^{16}$. Physicians in France, Germany, the United Kingdom, and the United States were randomly selected by members of a local research agency from the public lists of healthcare professionals and were screened for eligibility based on whether they were licensed physicians between 1975 and 2010, and actively managing 10 or more patients with gout per month. Each physician was asked to recruit 8 consecutive patients presenting with gout in his/her office. Included patients had a diagnosis of gout, had completed patient-reported data, and had to have been taking conventional ULT for $\geq 3$ months.

Data collection. In addition to in-depth interviews with each participating physician, physician workload questionnaires were collected that documented the number of gout or other patients on 5 consecutive days. Physicians also submitted an extensive patient record form for each patient that included the most recent SUA level and the number of flares in the previous 12 months. It also included whether the patient had any cardiovascular, autoimmune, gastrointestinal, renal, or other concomitant conditions. Patients were asked to complete a patient self-completion record containing the EQ-5D-3L and Patient Reported Outcomes Measurement Information System (PROMIS) Health Assessment Questionnaire-Disability Index (HAQ-DI) as well as the Work Productivity and Activity Impairment (WPAI) questionnaire. Only employed patients could provide data on work impairment, whereas all patients could provide data on activity impairment and HRQOL. Patients with SUA levels $>6.0 \mathrm{mg} / \mathrm{dl}$ (> $360 \mu \mathrm{mol} / \mathrm{l}$ ) or $\geq 2$ flares were designated as having gout that was inadequately controlled; those with SUA levels $\leq 6.0 \mathrm{mg} / \mathrm{dl}(\leq 360 \mu \mathrm{mol} / \mathrm{l})$ and 0 flares were designated as having gout that was adequately controlled. Patients with SUA $\leq 6.0 \mathrm{mg} / \mathrm{dl}$ $(\leq 360 \mu \mathrm{mol} / \mathrm{l})$ and only 1 flare fell outside of these 2 defined groups and were not included in the analysis.

EQ-5D-3L is a generic multiattribute health state classification system by which HRQOL is assessed in 5 dimensions: mobility, self-care, usual activities, pain/discomfort, and anxiety/depression ${ }^{17,18}$. Each dimension is evaluated using 3 levels: no problems, some problems, and severe problems. Responses to these 5 dimensions are converted into 1 of 243 different EQ-5D health state descriptions, which range from no problems on all 5 dimensions (11111) to severe/extreme problems on all 5 dimensions (33333). The scoring algorithm then converts the responses into a health utility specific to the individual's health state. In addition, a visual analog scale is used to have the individual rate their health state on a scale from $0-100$, with 0 being the worst health state imaginable and 100 being the best health state imaginable. The PROMIS HAQ has 20 items in 8 categories (dressing and grooming, hygiene, arising, reach, eating, grip, walking, common daily activities) ${ }^{19}$. The WPAI questionnaire consists of 6 questions: Q1, currently employed; Q2, hours missed owing to health problems; Q3, hours missed for other reasons; Q4, hours actually worked; Q5, degree that health affected productivity while working (using a 0-10 Likert scale); Q6, degree that health affected productivity in regular unpaid activities ( 0 to 10 Likert scale $)^{20}$. The recall period for Q2 to Q6 is 7 days.

Results for the EQ-5D domains are presented as the percentage of patients reporting no problem performing an activity, no pain or discomfort, no anxiety or depression; or reporting some problems, pain or discomfort, anxiety or depression; or reporting severe problems, extreme pain or discomfort, anxiety or depression. $\mathrm{P}$ values represent the difference between patients with controlled and uncontrolled gout across the 3 groups of each domain. Mean summary scores and $95 \% \mathrm{CI}$ are also presented, with lower scores indicating greater problems. For the PROMIS HAQ domains that inquire about the ability to perform an activity, results are presented as the sum of the percentage of patients reporting little difficulty, some difficulty, or much difficulty performing the activity, plus those unable to do the activity. Questions that inquired whether a patient needed, for example, a cane/crutches/walker/wheelchair, etc., are presented as the percentage of patients who answered "yes." $\mathrm{P}$ values represent the difference between controlled and uncontrolled gout cases across the 5 groups of difficulty (no, little, some, and much difficulty, and unable to do the activity) or in the percentage of patients who answered "yes." The PROMIS HAQ scores were calculated as described, with higher scores indicating greater difficulties ${ }^{21}$. Four main outcomes were generated from the WPAI questionnaire (Appendix 1) ${ }^{15,16}$. Results of the Likert scale questions are reported as mean plus 95\% CI.

Statistical analysis. Any patients with missing values for a particular variable were removed from all analyses where that variable was used. However, those patients were still eligible for inclusion in other analyses. The base of patients was expected to vary from 1 variable to another owing to imperfect physician knowledge, patients not wishing to answer the question, etc. Statistical differences between patients with uncontrolled and adequately controlled gout were assessed using t-tests or Mann-Whitney tests for continuous data and chi-squared or Fisher's exact tests for categorical data, depending on the distribution of the outcome variable and/or subgroup variable. No adjustments for multiplicity were made because of the exploratory nature of the analysis.

\section{RESULTS}

Patients. A total of 440 physicians were recruited from France (83), Germany (78), the United Kingdom (82), and the United States (197). Of the 1204 patients with gout receiving ULT included in this analysis, $836(69.4 \%)$ experienced inadequate control, whereas only $368(30.6 \%)$ had adequate control while taking their current ULT (Table 1). The time since diagnosis of gout was significantly less for

Personal non-commercial use only. The Journal of Rheumatology Copyright @ 2016. All rights reserved. 
Table 1. Baseline patient characteristics.

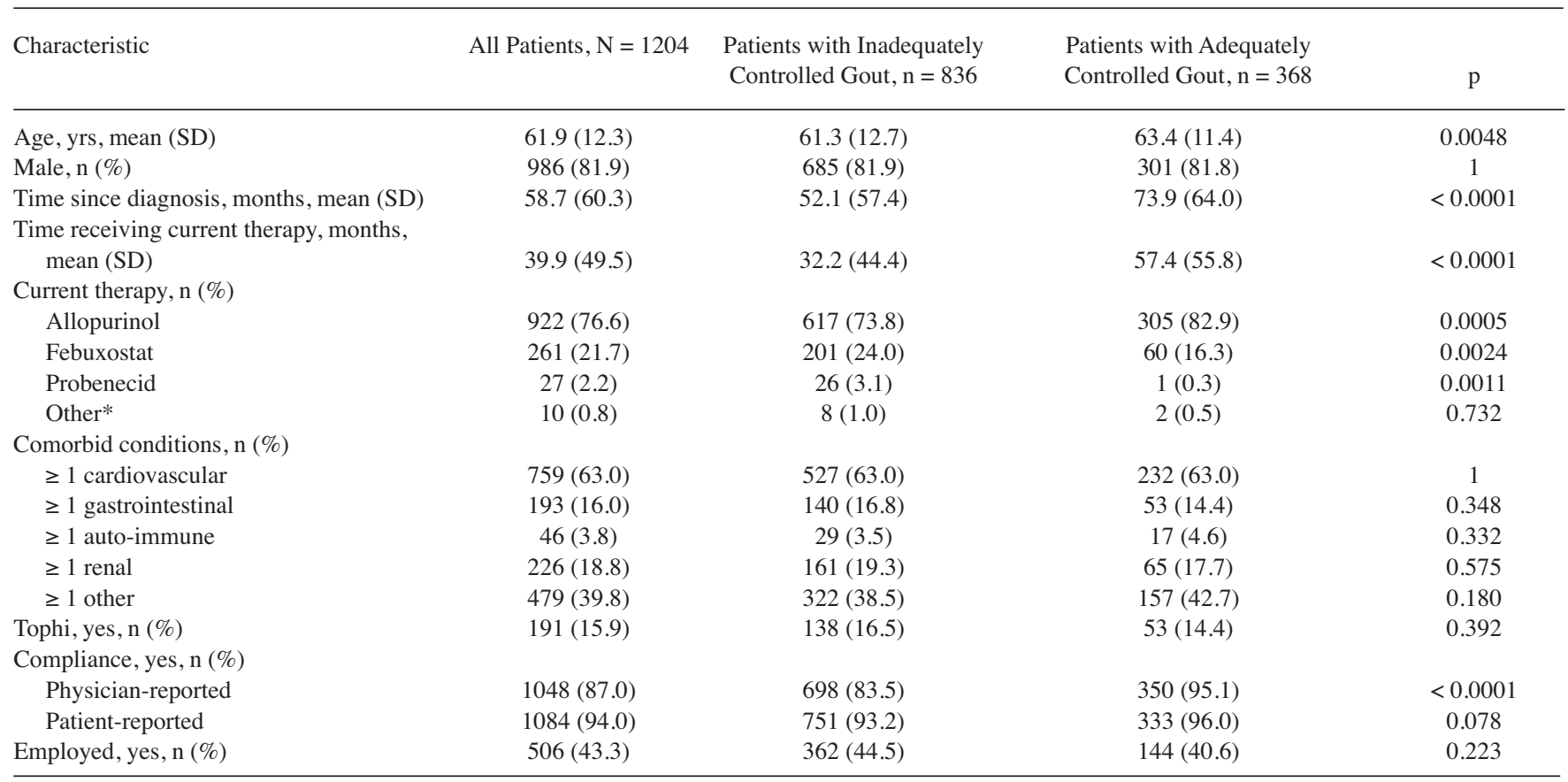

* "Other" includes benzbromarone, sulfinpyrazone, and pegloticase. For patients with inadequately controlled gout: serum uric acid (sUA) $>6$ mg/dl ( $>357$ $\mu \mathrm{mol} / \mathrm{l})$ or $\geq 2$ flares. For patients with adequately controlled gout: sUA $\leq 6 \mathrm{mg} / \mathrm{dl}(\leq 357 \mu \mathrm{mol} / \mathrm{l})$ and 0 flares.

patients with uncontrolled gout than for patients with controlled gout (average 52 vs 74 months, $\mathrm{p}<0.0001$ ). Although all patients, on average, were treated for more than 39 months with a ULT, the time of taking current ULT therapy was less for patients with uncontrolled gout (32 vs 57 months, $\mathrm{p}<0.0001)$. Current ULT for most patients was allopurinol (76.6\%), followed by febuxostat $(21.7 \%)$ and probenecid $(2.2 \%)$. More patients with adequately controlled gout than inadequately controlled gout were taking allopurinol $(82.9 \%$ vs $73.8 \%, \mathrm{p}<0.001)$, while fewer were taking febuxostat $(16.3 \%$ vs $24.0 \%, \mathrm{p}=0.002)$, or probenecid $(0.3 \%$ vs $3.1 \%, p=0.001)$. Sixty-three percent of patients had cardiovascular disease, with no difference between the groups. There also were no significant differences between groups in the percentage of patients with gastrointestinal, autoimmune, renal, or other comorbidities or in the percentage of patients with tophi. Patients with the greatest disease severity (i.e., inadequately controlled with tophi) experienced on average 2.2 flares a year and were more likely to have a cardiovascular condition $(71.0 \%$ vs $62.0 \%, \mathrm{p}=$ $0.040)$, an autoimmune condition $(7.3 \%$ vs $3.4 \%, p=0.034)$, or a renal condition $(37.0 \%$ vs $16.4 \%, \mathrm{p}<0.001)$ compared with patients with adequately controlled gout and patients with inadequately controlled gout without tophi, combined. Overall, $43.3 \%$ of patients were employed, with no significant difference between groups.

HRQOL. A greater percentage of patients with inadequately controlled gout than those with adequately controlled gout had problems performing the activities (mobility, self-care, and usual activities) of the EQ-5D domains, and more also had pain/discomfort and anxiety/depression (Figure 1A). The most prevalent problem was "pain/discomfort" while the least prevalent was "self-care." Significantly more patients with inadequately controlled gout had the problems than did patients with adequately controlled gout for all domains ( $\mathrm{p}<$ 0.0001 for each), while the total EQ-5D utility score was also significantly lower for patients with inadequately controlled gout (difference: $-0.087 ; \mathrm{p}<0.001$; Table 2 ). When patients were asked to rate how good or bad their health was today on a scale of 0 to 100 , patients with inadequately controlled gout rated themselves lower [mean $(95 \%$ CI), 69.2 (67.8-70.5)] than did patients with adequately controlled gout [78.1 (76.3-79.9); $\mathrm{p}<0.001]$.

Patients who had inadequately controlled gout also had significantly more difficulty performing most of the activities of the PROMIS HAQ domains (Table 3 ). The 2 exceptions where there was no difference between the groups were the need for a buttonhook/zipper pull/gadget or help to get dressed and the need for a long-handled appliance or help to reach something. The PROMIS HAQ score was significantly worse for patients with inadequately controlled gout compared with patients with adequately controlled gout (difference 6.302; $\mathrm{p}<0.001$; Figure 1B).

Work productivity and activity impairment. Overall, patients missed an average of $3.6 \%$ of their work time because of gout. Patients with inadequately controlled gout missed

Personal non-commercial use only. The Journal of Rheumatology Copyright $@$ 2016 . All rights reserved. 
A.

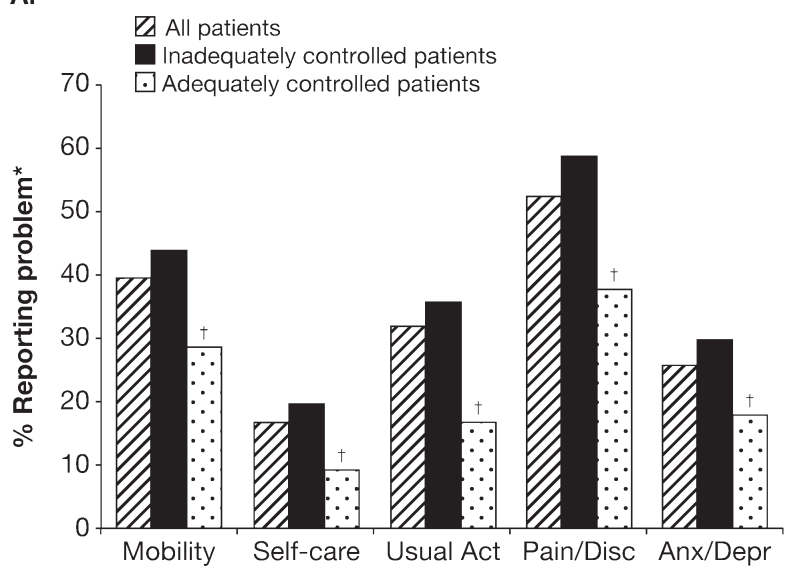

B.

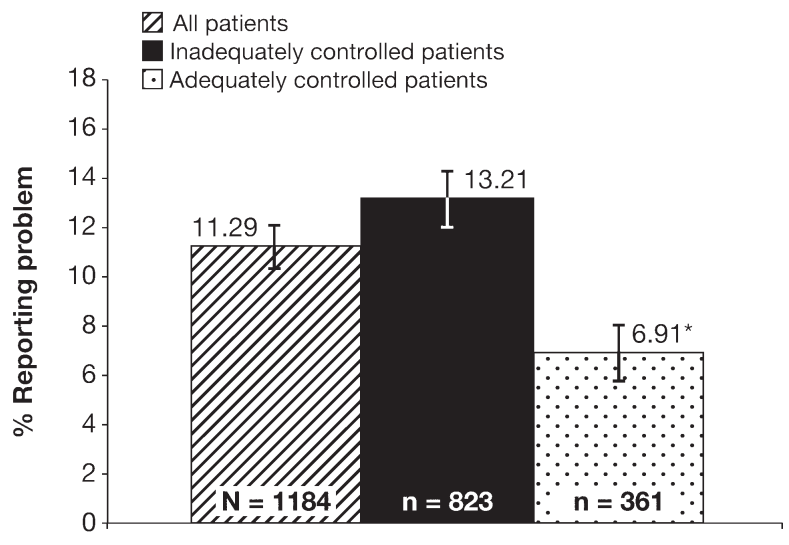

Figure 1. Percentage of patients reporting problems performing EQ-5D domain activities or PROMIS HAQ score - patients with inadequately vs adequately controlled gout. A. *Defined for mobility as the sum of percentage of patients with some problems in walking about + percentage confined to bed; for self-care, as sum of percentage with some problems + percentage unable to wash or dress; for usual activities (act), as sum of percentage with some problems + percentage unable to perform usual activities; for pain/discomfort (disc), sum of percentage with moderate + percentage with extreme pain or discomfort; for anxiety/depression (anx/depr), sum of percentage moderately + percentage extremely anxious or depressed. $\dagger \mathrm{p}<$ 0.001 vs patients with inadequately controlled gout across the 3 groups of each domain (no, some, or extreme problem). B. Data are mean ( $95 \%$ CI). $* \mathrm{p}<0.001$ vs patients with inadequately controlled gout.

Table 2. EQ-5D domain summary scores by prevalence - patients with inadequately vs adequately controlled gout.

\begin{tabular}{|c|c|c|c|}
\hline Domain & $\begin{array}{c}\text { Percent Reporti } \\
\text { Patients with } \\
\text { Inadequately } \\
\text { Controlled } \\
\text { Gout, } \mathrm{N}=836\end{array}$ & $\begin{array}{l}\text { No/Some/Severe* } \\
\text { Patients with } \\
\text { Adequately } \\
\text { Controlled Gout, } \\
\quad \mathrm{N}=368\end{array}$ & $\mathrm{p}^{\dagger}$ \\
\hline Pain/discomfort & $41.0 / 52.3 / 6.7$ & $62.2 / 36.1 / 1.7$ & $<0.0001$ \\
\hline Mobility & $55.4 / 44.2 / 0.4$ & $70.8 / 29.2 / 0.0$ & $<0.0001$ \\
\hline Usual activities & $61.3 / 36.3 / 2.4$ & $81.9 / 17.2 / 0.8$ & $<0.0001$ \\
\hline Anxiety/depression & $70.0 / 27.5 / 2.4$ & $82.2 / 16.2 / 1.7$ & $<0.0001$ \\
\hline Self-care & $79.8 / 19.7 / 0.5$ & $90.5 / 9.5 / 0.0$ & $<0.0001$ \\
\hline
\end{tabular}

* No problems, some problems, severe problems. ${ }^{\dagger} \mathrm{p}$ values represent the difference between patients with inadequately controlled gout and adequately controlled gout across the 3 groups of each domain.

significantly more time (4.5\%) than did those with adequately controlled gout $(1.3 \% ; \mathrm{p}<0.001$; Figure $2 \mathrm{~A})$. They were also significantly more impaired because of gout while working ( $19.1 \%$ vs $5.2 \%$; $<<0.001$; Figure $2 \mathrm{~B})$, had greater overall work impairment due to gout (20.4\% vs $5.6 \%$; p $<0.001$; Figure $2 \mathrm{C}$ ), and had greater activity impairment due to gout (20.3\% vs 5.3\%; $p<0.001$; Figure 2D). During the previous 7 days, patients with inadequately controlled gout missed significantly more hours of work (average $1.6 \mathrm{~h}$ ) than did patients with adequately controlled gout $(0.4 \mathrm{~h} ; \mathrm{p}<0.001$; Table 4). However, hours missed for other reasons (2.8 vs 3.4 $\mathrm{h} ; \mathrm{p}=0.458)$ and hours actually worked (35.0 vs 31.5 hours; $\mathrm{p}=0.174)$ were not different between the groups.

\section{DISCUSSION}

Consistent with published literature, the results of our study show that relatively few cases of gout are adequately controlled by conventional ULT. The recent report by Juraschek and colleagues on ULT and uric acid levels suggested that SUA levels were above target in half of those receiving ULT and two-thirds of those with an indication for $\mathrm{ULT}^{22}$. In our real-world survey, despite more than 39 months of treatment, even fewer patients - less than one-third achieved complete disease control. Patients who had inadequately controlled gout with tophi experienced more flares a year and were more likely to have comorbid conditions compared with those with adequately controlled gout and inadequately controlled gout without tophi, combined.

Patients with inadequately controlled gout while taking their current ULT reported significantly worse functioning and HRQOL compared with patients who had controlled gout, as measured by EQ-5D and PROMIS HAQ scales. They also missed more work, had greater impairment in work productivity, and more activity impairment, as measured by the WPAI questionnaire, than did patients with adequately controlled gout. These results are consistent with those from previous longitudinal observational studies ${ }^{13,14,15}$.

Despite the availability of multiple treatment guidelines ${ }^{23,24,25}$, gout in the majority of patients remains poorly controlled. Current data suggest that strategies for improving management of patients with gout are needed because very few patients are being treated effectively.

There are limitations to our study. Respondent physicians were requested to collect data on a series of consecutive patients to avoid selection bias; this was contingent upon the integrity of the participating respondent rather than formalized source verification procedures. Similarly, diagnosis in the patient group was based primarily on the judgment and diagnostic skills of the respondent physician rather than on a formalized diagnostic checklist. The DSP is

Personal non-commercial use only. The Journal of Rheumatology Copyright @ 2016. All rights reserved 
Table 3. Percentage of patients reporting difficulty performing PROMIS HAQ domain activities - patients with inadequately vs adequately controlled gout.

Activities

$\%$ Reporting No/Little/Some/Much Difficulty/Unable to do

Patients with Inadequately

Controlled Gout, $\mathrm{N}=836$
Patients with Adequately

Controlled Gout, N = 368 $\mathrm{p} *$
$<0.0001$

$<0.0001$

$<0.0001$

$<0.0001$

$<0.0001$

$<0.0001$

0.0002

$<0.0001$

$<0.0001$

$<0.0001$

$<0.0001$

$<0.0001$

$<0.0001$

$<0.0001$

$<0.0001$

0.0001

$<0.0001$

$<0.0001$

$<0.0001$

$<0.0001$

$<0.0001$

0.001

0.152

0.057

Need a long-handled appliance or help to reach something, \% yes

16.2

7.2

4.6

5.7
6.4

2.5

2.8

3.1

* P value represents the difference between patients with controlled and uncontrolled gout across the 5 groups of difficulty or in the percent responding "yes." PROMIS: Patient Reported Outcomes Measurement Information System; HAQ: Health Assessment Questionnaire.

a cross-sectional database, so it cannot be used to demonstrate cause and effect. In some cases (e.g., patients with the need to durably improve signs and symptoms of gout, including palpable and visible tophi), target SUA levels of $\geq$ $5 \mathrm{mg} / \mathrm{dl}(\geq 300 \mu \mathrm{mol} / \mathrm{l})$ may constitute an "inadequately controlled" case.

Gout control remains a considerable clinical challenge, with less than one-third of patients achieving adequate control in this analysis. This challenge is augmented by the observation that daily function, quality of life, and work productivity are significantly worse in those patients with gout whose disease is inadequately controlled. This not only affects the patients themselves, but also imparts societal and work-based effects, leading to substantial economic burden. Improved gout treatment strategies remain a critical unmet need; steps to enhance the control of hyperuricemia may lead to gout symptom improvements and a resultant increase in HRQOL and work productivity.

\section{ACKNOWLEDGMENT}

Under the direction of the authors, editorial support was provided by Tom Claus of PAREXEL and funded by AstraZeneca.

\section{REFERENCES}

1. Doghramji PP, Wortmann RL. Hyperuricemia and gout: new concepts in diagnosis and management. Postgrad Med 2012;124:98-109.

2. Boss GR, Seegmiller JE. Hyperuricemia and gout. Classification, complications and management. N Engl J Med 1979;300:1459-68.

3. Kannangara DR, Ramasamy SN, Indraratna PL, Stocker SL, Graham GG, Jones G, et al. Fractional clearance of urate: validation of measurement in spot-urine samples in healthy subjects and gouty patients. Arthritis Res Ther 2012;14:R189.

4. Terkeltaub R, Edwards NL. Clinical features and natural course. In: Terkeltaub R, Edwards NL, editors. Gout: diagnosis and management of gouty arthritis and hyperuricemia. Durant, Oklahoma: Professional Communications Inc.; 2013.

5. Zhu Y, Pandya BJ, Choi HK. Comorbidities of gout and hyperuricemia in the US general population: NHANES 2007-2008. Am J Med 2012;125:679-87.

6. Khanna PP, Nuki G, Bardin T, Tausche AK, Forsythe A, Goren A, et al. Tophi and frequent gout flares are associated with impairments to quality of life, productivity, and increased healthcare resource use: results from a cross-sectional survey. Health Qual Life Outcomes 2012;10:117.

7. Singh JA, Sarkin A, Shieh M, Khanna D, Terkeltaub R, Lee SJ, et al. Health care utilization in patients with gout. Semin Arthritis Rheum 2011;40:501-11.

8. Smith EU, Diaz-Torne C, Perez-Ruiz F, March LM. Epidemiology of gout: an update. Best Pract Res Clin Rheumatol 2010;24:811-27.

Personal non-commercial use only. The Journal of Rheumatology Copyright @ 2016 . All rights reserved. 

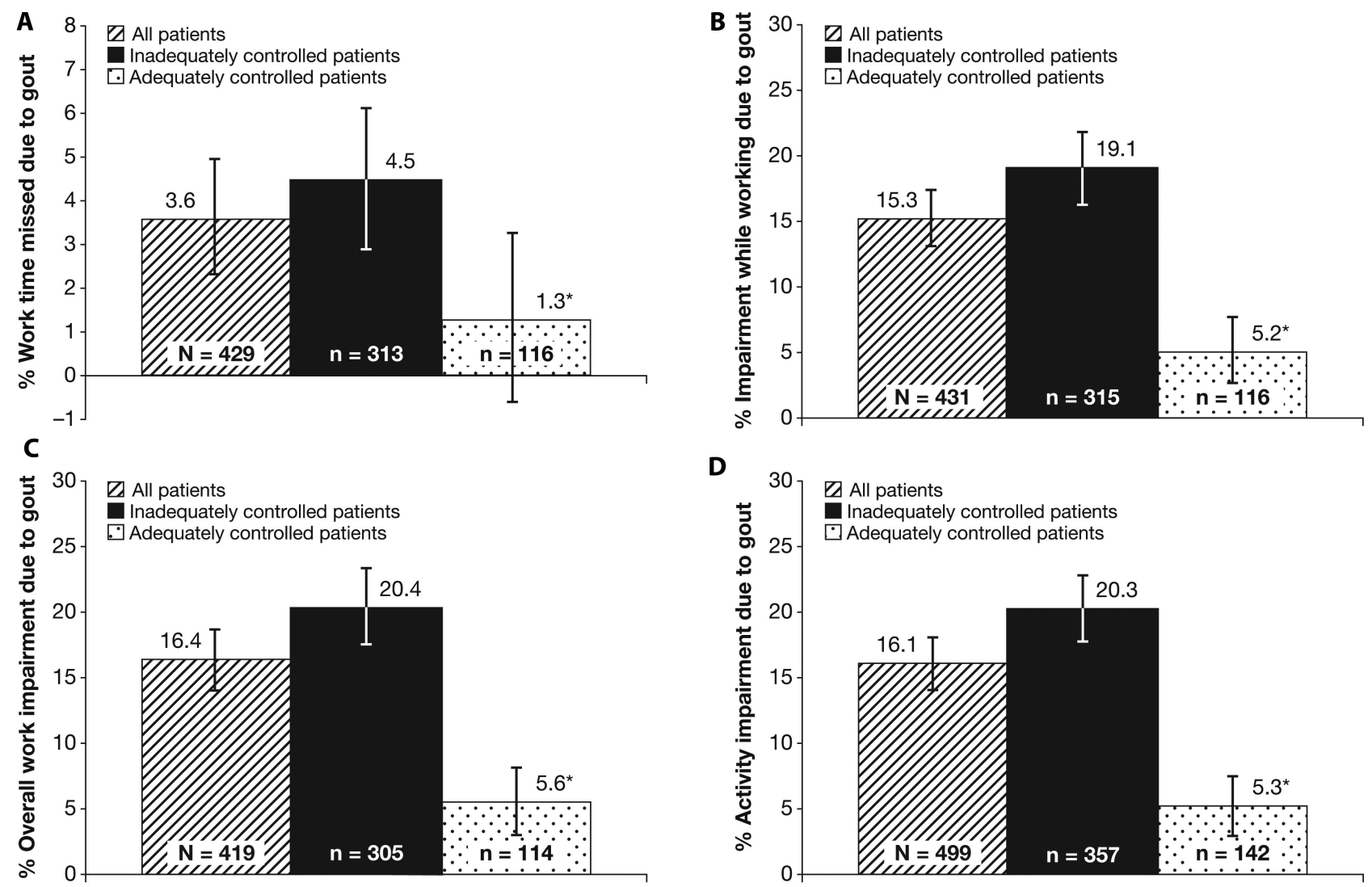

Figure 2. Percentage of (A) work time missed because of gout, (B) impairment while working due to gout, (C) overall work impairment due to gout, and (D) activity impairment due to gout - patients with inadequately controlled vs adequately controlled gout. Data are mean $(95 \% \mathrm{CI}) . * \mathrm{p}<0.001$ vs patients with inadequately controlled gout.

Table 4. Work productivity and daily activities in the past 7 days - patients with inadequately controlled vs adequately controlled gout. Data are mean $(90 \% \mathrm{CI})$.

\begin{tabular}{lccc}
\hline & $\begin{array}{c}\text { Patients with Inadequately } \\
\text { Controlled Gout }\end{array}$ & $\begin{array}{c}\text { Patients with Adequately } \\
\text { Controlled Gout }\end{array}$ & $\mathrm{p}$ \\
\hline $\begin{array}{l}\text { Hours missed during past 7 days } \\
\text { because of gout problems }\end{array}$ & $1.6(1.0-2.3)$ & $0.4(-0.2-1.0)$ & 0.001 \\
$\begin{array}{l}\text { Hours missed during past 7 days for other } \\
\text { reasons (vacation, etc.) }\end{array}$ & $2.8(1.9-3.7)$ & $3.4(1.8-5.1)$ & 0.46 \\
$\begin{array}{l}\text { Hours actually worked during past 7 days } \\
\text { How gout affected productivity at work } \\
\quad \text { during past 7 days }\end{array}$ & $35.0(33.4-36.5)$ & $31.5(28.6-34.4)$ & 0.17 \\
$\begin{array}{l}\text { How gout affected daily activities (not work) } \\
\text { during past 7 days }\end{array}$ & $1.91(1.64-2.18)$ & $0.52(0.27-0.77)$ & $<0.001$ \\
\hline
\end{tabular}

9. Wertheimer A, Morlock R, Becker MA. A revised estimate of the burden of illness of gout. Curr Ther Res Clin Exp 2013;75:1-4.

10. Perez-Ruiz F. Treating to target: a strategy to cure gout. Rheumatology 2009;48 Suppl 2:ii9-14.

11. Perez-Ruiz F, Herrero-Beites AM. Evaluation and treatment of gout as a chronic disease. Adv Ther 2012;29:935-46.

12. Roddy E, Doherty M. Epidemiology of gout. Arthritis Res Ther 2010;12:223.

13. DiBonaventura M, Andrews LM, Yadao AM, Kahler KH. The effect of gout on health-related quality of life, work productivity, resource use and clinical outcomes among patients with hypertension. Expert Rev Pharmacoecon Outcomes Res 2012;12:821-9.

14. Khanna PP, Nuki G, Bardin T, Tausche AK, Forsythe A, Goren A, et al. Tophi and frequent gout flares are associated with impairments to quality of life, productivity, and increased healthcare resource use: results from a cross-sectional survey. Health Qual Life Outcomes 2012;10:117

15. Saseen JJ, Agashivala N, Allen RR, Ghushchyan V, Yadao AM, Nair $\mathrm{KV}$. Comparison of patient characteristics and gout-related health-care resource utilization and costs in patients with frequent 
versus infrequent gouty arthritis attacks. Rheumatology 2012;51:2004-12.

16. Anderson P, Benford M, Harris N, Karavali M, Piercy J. Real-world physician and patient behaviour across countries: Disease-Specific Programmes - a means to understand. Curr Med Res Opin 2008;24:3063-72.

17. EuroQol-a new facility for the measurement of health-related quality of life. Health Policy 1990;16:199-208.

18. EuroQol. EQ-5D-3L user guide. Basic information on how to use the EQ-5D-3L instrument. EuroQol Org. [Internet. Accessed February 26, 2016]. Available from: www.euroqol.org/fileadmin/ user_upload/Documenten/PDF/Folders_Flyers/EQ-5D-3L_ UserGuide_2015.pdf

19. Fries JF, Spitz PW, Young DY. The dimensions of health outcomes: the health assessment questionnaire, disability and pain scales. J Rheumatol 1982;9:789-93.

20. Reilly MC, Zbrozek AS, Dukes EM. The validity and reproducibility of a work productivity and activity impairment instrument. Pharmacoeconomics 1993;4:353-65.
21. Ace Learning Company. HAQ Instructions (ARAMIS Site) Learning Ace Web site. [Internet. Accessed February 26, 2016]. Available from: www.learningace.com/doc/1555309/ 8f2cb45d41c6d88f5cb786a2b3bc1fa1/ haq-instructions-aramis-site-2-14-08

22. Juraschek SP, Kovell LC, Miller ER III, Gelber AC. Gout, urate lowering therapy and uric acid levels among US adults. Arthritis Care Res 2014;67:588-92.

23. Hamburger M, Baraf HS, Adamson TC III, Basile J, Bass L, Cole B, et al. 2011 recommendations for the diagnosis and management of gout and hyperuricemia. Postgrad Med 2011;123 (6 Suppl 1):3-36.

24. Jordan KM, Cameron JS, Snaith M, Zhang W, Doherty M, Seckl J, et al. British Society for Rheumatology and British Health Professionals in Rheumatology guideline for the management of gout. Rheumatology 2007;46:1372-4.

25. Khanna D, Fitzgerald JD, Khanna PP, Bae S, Singh MK, Neogi T, et al. 2012 American College of Rheumatology guidelines for management of gout. Part 1: systematic nonpharmacologic and pharmacologic therapeutic approaches to hyperuricemia. Arthritis Care Res 2012;64:1431-46.

APPENDIX 1. Calculation of WPAI questionnaire outcomes.

\begin{tabular}{|c|c|}
\hline Outcome & Equation* \\
\hline Percentage of work time missed because of health & $\begin{array}{l}\mathrm{Q} 2 /(\mathrm{Q} 2+\mathrm{Q} 4) \text { for those who were currently } \\
\text { employed }\end{array}$ \\
\hline Percentage of impairment while working because of health & $\begin{array}{l}\text { Q5/10 for those who were currently employed and } \\
\text { actually worked in the previous } 7 \text { days }\end{array}$ \\
\hline Percentage of overall work impairment due to health & $\begin{array}{l}\mathrm{Q} 2 /(\mathrm{Q} 2+\mathrm{Q} 4)+((1-\mathrm{Q} 2 /(\mathrm{Q} 2+\mathrm{Q} 4)) \times(\mathrm{Q} 5 / 10)) \\
\text { for those who were currently employed }\end{array}$ \\
\hline Percentage of activity impairment due to health & Q6/10 for all respondents \\
\hline
\end{tabular}

* Results expressed in percentages by multiplying each score by 100. WPAI: Work Productivity and Activity Impairment; Q: question; Q1: currently employed; Q2: hours missed due to health problems; Q3: hours missed for other reasons; Q4: hours actually worked; Q5: degree that health affected productivity while working (using a 0-10 Likert scale); Q6: degree that health affected productivity in regular unpaid activities (0-10 Likert scale). 